\title{
ALOE; BEYOND USE AS COSMETICS
}

\author{
By Kameju Pili, MD2 2006/2007
}

\section{INTRODUCTION}

Aloe is a succulent of the lily family. It is native to Africa and is widely distributed across the continent. Plant occurring Aloe species are vera and barbadensis

The aloe plant has fleshy leaves which can be extracted to:

1. Gel

2. latex

The gel is the leaf pulp or mucilage, a thin clear jelly-like substance obtained from the parenchymal tissue that makes up the inner portion of the leaves.'

Aloe latex, or "aloe juice," is a bitter yellow exudate from the pericyclic tubules just beneath the outer skin of the leaves.

Aloe latex contains the anthraquinone glycosides aloin $A$ and $B$, which are potent laxatives ${ }^{2}$ and are used pharmaceutically

Aloe is widely used in cosmetics such as oils, lotions, soaps, shampoos and other beauty products. Many people are ignorant of how aloe can be used medicinally. Research has proven that aloe contains potent agents that aid in treating numerous ailments, with minimal side effects. It also contains vitamins and amino acids and is therefore nutritious product for humans and animals.

\section{ALOE HISTORY}

Aloe has been used from time immemorial.

The "Papyrus ebers", an Egyptian document written around B.C.E. 1550 gave twelve formulae for mixing Aloe products with other agents to treat both internal and external human disorders.

In Greek pharmacology, the plant was first mentioned by Celsius (B.C. 25-50 A.D.), but his comments were limited to its power as a purgative.

In 41-68 AD; Discoroides, the master of Roman pharmacology gave the first detailed description of the plant and its use as a laxative, detoxifier, treatment for boils, bruises, tonsils, gums and eyes.

In 700-800 A.D; Chinese "Materia Medicas", first use of Aloe in China notes Aloe's value as a sinus treatment and treatment of fever and convulsions in children. Also re-confirmed use as a treatment for skin diseases.
The Bible has references of aloe use, e.g. in Numbers 24:6, Psalms 45:8, Proverbs 7:17 and John 19:39

\section{Aloe Contents}

Aloe contains at least 6 antiseptic agents:

1. Lupeol,

2. Salicylic acid,

3. Urea nitrogen,

4. Cinnamonic acid,

5. Phenols, and

6. Sulphur

All of these substances are recognized as antiseptics because they kill or control moulds, bacteria, and viruses. The lupeol and salicylic acid (aloe by products) explain why it is an effective pain-killer.

Aloe contains at least 3 anti-inflammatory fatty acids:

1. cholesterol,

2. campersterol

3. B-sitosterol

This explains why it is a highly effective treatment for burns, cuts, scrapes, abrasions, allergic reactions, ulcers and many inflammatory conditions of the digestive system and internal organs

B-sitosterol is also a powerful anti-cholestromatic which helps to lower harmful cholesterol levels, helping to explain its many benefits for patients with cardiac disorders.

Aloe contains at least 23 polypeptides which are immune stimulators. This explains why Aloe may ameliorate a broad spectrum of immune system diseases and disorders, such as HIV and AIDS.

The polypeptides, plus the anti-tumor agents Aloe emodin and Aloe lectins, explains its ability to control cancer.

\section{Diseases treated by Aloe products}

\section{Aloe and the Immune System}

Scientific evidence suggests that aloe gel contains substances that are active both in stimulating phagocytosis as well as stimulating formation of antibodies.

In one study, aloe fractions were shown to increase phagocytosis when injected in guinea pigs. ${ }^{3}$ 


\section{Aloe and HIV and AIDS}

Aloe vera contains Gluco mannan, a complex polysaccharide composed largely of the sugar mannose. In a 1991 study in Molecular Biotherapy, HIV1 infected cells were treated invitro with the mannose extract. Virus reproduction decreased by as much as $30 \%$ by Aloe Vera viral load was reduced. Spread of the virus from the infected cells was suppressed. Viability of infected cells was also increased.

According to the Journal of Advancement in Medicine, the Aloe vera gel also proved to be an effective part of a nutritional support program for HIV infected patients. For 4 months, 29 patients were given 100\% pure Aloe vera gel (5 ounces, 4 times daily) along with an essential fatty acid supplement and another supplement containing vitamins, minerals and amino acids. Patients were told to continue with their normal diet and not to take other supplements.

After 90 days all of the patients had fewer occurrences of opportunistic infections, thrush, fatigue and diarrhoea, as well as increased white blood cell counts, particularly $\mathrm{T} 4$ helper cells.

\section{Anti-Cancer Effects}

In a study conducted in Istanbul Aloe vera leaf pulp extract was used against Ehrlich ascites tumours in mice 4 .

The animals were separated into five groups:

I - healthy control,

I - Untreated tumour control,

III - experiment 1 (extract given before tumour inoculation),

IV - experiment 2 (extract given with tumour inoculation) and

$V$ - experiment 3 (extract given after tumour inoculation).

Ehrlich ascites tumours $(0.33 \mathrm{ml})$ were injected subcutaneously into groups $\|-\mathrm{V}$.

Aloe extract was injected at $55 \mathrm{mg}$ protein/ $\mathrm{kg}$, twice a week for 21 days.

Tumour size, thymus and spleen weights were measured, as well as leucocyte count, tumour necrosis factor-alpha and sialic acid as tumour markers. Results:

- The best inhibitory effect on tumour growth was obtained with the extract given prophylactically before tumour implantation (experiment 1)
- Aloe extract also regressed tumour sizes when given simultaneously with (experiment 2), or therapeutically after (experiment 3), tumour implantation.

- Serum sialic acid and tumour necrosis factoralpha levels, chosen as tumour markers, which were raised in the tumour control group, were significantly decreased by the prophylactic administration of the extract.

- An increase in leucocyte count was seen in experiment 1 and 2 groups, along with lymphoid hyperplasia observed in spleen and thymus necroscopy.

- This suggests that the tumour preventive effect of Aloe could be due to its immunomodulatory activity.

According to the results, Aloe vera could be proposed as a prophylactic for cancer prevention.

\section{Atherosclerosis and Heart Diseases}

In 1985, a study was reported in Angiology, The Journal of Vascular Diseases. ${ }^{5}$, five thousand patients, aged 3565 , were selected to be in this five-year study.

To qualify, each person was required to have proven heart disease with angina pectoris, documented by EKG.

Fresh Aloe vera gels (100 grams) as well as Isabgol (which increases the bulk of faeces) were added to the patients diets. During the five years of the study, all five thousand patients were examined weekly.

\section{Results}

- More than 93\% of the patients experienced subjective and objective improvement in symptoms, blood chemistries, and EKG's.

- There was a marked reduction in total lipids, total serum cholesterol, serum triglycerides, in fasting and post-meal blood sugar levels in diabetics, and an increase in High Density Lipoproteins (HDL).

- The angina patients in the study lowered their harmful Low Density Lipoprotein (LDL) cholesterols and triglycerides by an astounding 40-80\% and 20-70\%, respectively, with no side effects! 
- Patients with high blood pressure were able to cut their medications in half.

- All of the diabetics were originally taking oral drugs. However, gradually their blood sugar levels came down to normal.

- After taking Aloe vera for two months, 94\% of the 3,167 diabetics stopped their oral hypoglycemic drugs and controlled their blood sugar with diet alone.

- Not one patient experienced a hypoglycemic episode during the study

\section{Anti-Diabetic effects}

A laboratory experiment at the Univ. of Zimbabwe was carried out on both normal and diabetic rats to investigate the effect of Aloe excelsa powder on diabetics ${ }^{6}$.

Diabetes was induced in the rats by using streptozotocin. Blood samples were taken from both the normal and diabetic rats and the sugar levels measured. The subjects were a total of 280 male albino rats weighing $250 \mathrm{~g}$ on average.

\section{Results}

The Aloe excelsa powder produced a dose-dependent reduction in the blood glucose levels. It compared well with the effect of insulin.

This study shows that Aloe excelsa has significant activity on glucose levels in rats and this, therefore, confirms the claims by traditional healers that this plant has anti-diabetic effects.

\section{Anti-Viral effects}

A study was conducted in the USA ${ }^{7}$ to determine the extent of antiviral activity present in a number of plant extracts.

Aloe emodin was tested against infectivity of Herpes simplex virus type 1 and type 2, varicella-zoster virus, pseudorabies virus, influenza virus, adenovirus, and rhinovirus.

\section{Results}

Aloe emodin inactivated all of the viruses tested except adenovirus and rhinovirus.

Electron microscopic examination of anthraquinonetreated herpes simplex virus demonstrated that the envelopes were partially disrupted.

These results show that anthraquinones extracted from Aloe emodin are directly virucidal to enveloped viruses

\section{Anti-fungal effects}

A study was done in India ${ }^{8}$ to investigate the anti fungal activity of anthraquinone derivatives of Rheum emodi.

Rhein, physcion, Aloe emodin and chrysophanol isolated from Rheum emodi rhizomes exhibited antifungal activity against Candida albicans, Cryptococcus neoformans, Trichophyton mentagrophytes and Aspergillus fumigatus

\section{Long term effects of Aloe ingestion}

A study was done in Texas, USA with the objective of initiating a systematic and scientific investigation of the effects of long-term aloe ingestion on laboratory rats 9 . A total of 360 rats were divided into four groups:

- Group 1 (control) was fed a semi-synthetic diet without aloe;

- Group 2 was fed a diet containing a 1\% freezedried aloe filet;

- Group 3 was fed a diet containing a $1 \%$ charcoal-processed, freeze-dried aloe filet; and

- Group 4 was given whole leaf aloe $(0.02 \%)$ in drinking water.

\section{Results}

Aloe ingestion, both crude and processed, was shown to extend average life span by $10 \%$ and slowed the mortality rate doubling time. Group 2 and 3 showed a lower incidence of atrial thrombosis than Group 1. Group 2 showed a significantly lower incidence of fatal chronic nephropathy and occurrence of multiple causes of death compared to the control group. All groups ingesting aloe showed a slightly lower incidence of fatal leukemia. No adverse, toxic effects were found with the ingestion of Aloe vera.

\section{Effects of Aloe vera gel to burn wound healing}

In a study conducted in Thailand, 27 patients with partial thickness burn wound were treated with Aloe vera gel compared with vaseline gauze ${ }^{10}$.

\section{Results}

The average time of healing in the aloe gel area was 11.9 days compared to 18.2 days for the vaseline gauze treated wound. Statistical analysis by using t-test and the value of $P<0.002$ was statistically significant.

Histologically, there was early epithelialization in the Aloe vera gel treated area.

Only some minor adverse effects, such as discomfort and pain were encountered. 


\section{Conclusion}

This study showed the effectiveness of Aloe vera gel on a partial thickness burn wound, and it might be beneficial to do further trials on burn wounds

\section{Effects on the gastrointestinal tract}

A study was conducted in California, USA to investigate effects of Aloe vera on the gastro intestinal tract of normal people. Aloe vera was found to have several measurable effects ${ }^{11}$.

\section{Results}

There was a faster movement of food through the bowel with better protein digestion and absorption. There was an increase in water in the stool making it bulkier, and a normalisation of stool bacteria, where there had previously been high levels of yeasts in some of the subjects.

\section{Contraceptive effect of Aloe products}

A study was conducted in Missouri, USA to test the contraceptive effect of Aloe barbadensis and zinc acetate $^{12}$.

Aloe barbadensis at concentrations of 7.5\% and 10\% proved to be spermicidal due to the multiple micro elements (boron, barium, calcium, chromium) which were toxic to the sperm tail causing instant immobilization.

Zinc acetate was spermicidal due to the acetate which may decrease oxygen utilization by sperm. It was also virucidal in vitro while aloe was not.

The two compounds did not irritate or cause ulceration of rabbit vaginal epithelium.

These results suggest the possibility of using the zinc acetate and Aloe barbadensis as a new, effective and safe vaginal contraceptive.

\section{Preparation of Aloe Nutrition Drink}

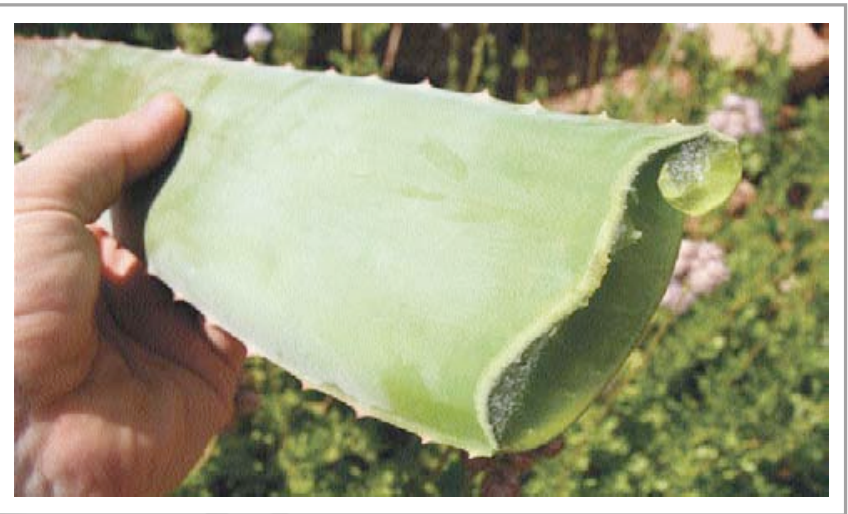

1. Cut a leaf from the lower part of the plant

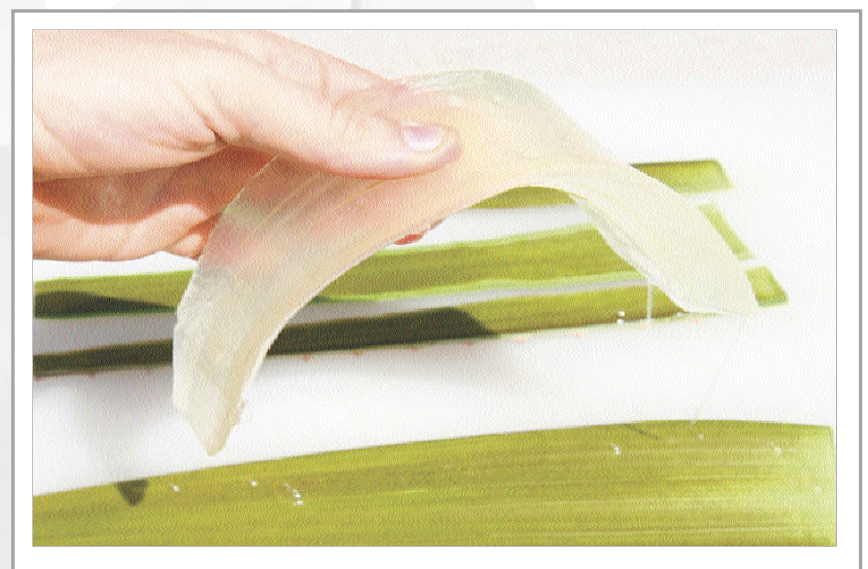

3. Carefully peel off the gel from the latex

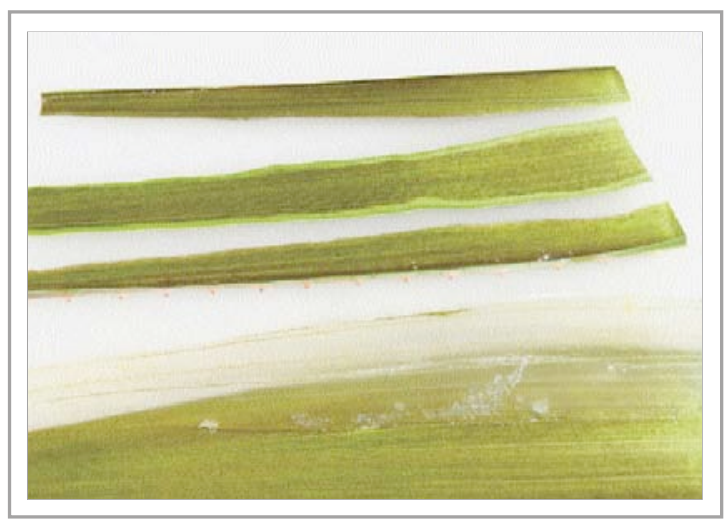

2. Cut away the upper thick green part (latex) to reveal the inner gel

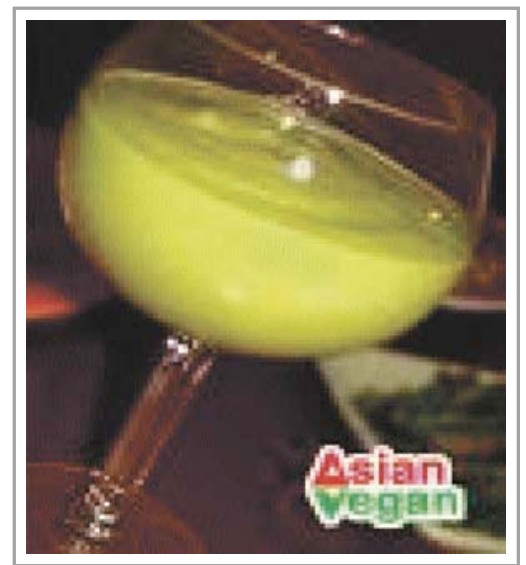

4. Blend the gel and add milk, sugar and/or honey to add flavor. The drink is ready for use 


\section{Side effects of Aloe extracts}

Aloe latex is a strong laxative and can cause severe cramping and diarrhea. Pregnant or breast feeding women should particularly avoid it. Diarrhea caused by the laxative effect of oral Aloe vera, can decrease the absorption of many drugs.

In rare cases, topical use of aloe produces a mild, allergic skin reaction with itching or rash; simply discontinue use. Do not apply aloe gel on open wounds

Use for diabetes should be with some caution. Preliminary studies suggest taking Aloe vera by mouth may lower blood glucose levels in people with diabetes who use glucose-lowering medication.

Overuse of Aloe vera juice can cause loss of potassium and may lead to toxicity when taken with the heart/blood pressure drugs digitoxin and digoxin or steroids such as methylprednisolone, and prednisone. It also may intensify the potassium-depleting effects of diuretics such as chlorothiazide, hydrocholorothiazide, and indapamide

\section{Aloe in Tanzania}

Aloe being a tropical plant grows in every region of Tanzania. No research has been carried out on aloe use for treatment of human diseases. There is little awareness of the wide variety of uses of aloe in treatment of diseases. There is little awareness of how to properly prepare aloe for use. It is currently used as a traditional medicine.

The Institute of Traditional Medicine at Muhimbili University of Health and Allied Sciences (MUHAS) has grown it and advocates its use by HIV positive people in whom it has shown to improve the state of health. The current medication for the above mentioned diseases which could also treated by aloe are expensive, inaccessible and produce many side effects.

\section{Conclusion and Recomendations}

Since aloe grows almost everywhere in Tanzania, it is easily accessible. Since aloe produces fewer side effects, it can be substituted for other drugs once proper dosages of aloe usage have been established.

Research should be conducted on aloe in order to learn how best to prepare, package and use it at appropriate dosages. Aloe should be grown on a large scale and exported to countries where it is not available

\section{REFERENCES}

1. Tyler V. The Honest Herbal: A Sensible Guide to the Use of Herbs and Related Remedies, Third Edition. Binghamton, NY: Pharmaceutical Products Press; 1993.

2. Tyler V. Herbs of Choice: The Therapeutic Use of Phytomedicinals. Binghamton, NY: Pharmaceutical Products Press; 1994.

3. Stepanova O.S, Prudnik N.Z, Solov'eva VP, Golovchenko G.A, Svischuk AA, Grin Erg Bg,Dubkova O.M: Chemical Composition and Biological activity of dry aloe leaves. Fiziol Akt Veshcheztva 9:94-97, 1977.

4. Akev N, Turkay G, Can A, Gurel A, Yildiz F, Yardibi H, Ekiz $\mathrm{EE}$, Uzun $\mathrm{H}$, Aloe vera leaf pulp extract versus Ehrlich ascites tumours in mice, European Journal of Cancer Prev.; 16(2):151-7 2007

5. O.P. Agarwal, Angiology, Atherosclerosis and Heart Disease, Journal of Vascular Diseases 36(8); 485-492, 1985.

6. Gundidza M, Masuku S, Humphrey G, Magwa ML. The effect of Aloe excelsa powder on normal and diabetic rats, Central African Journal of Medicine. ;51(11-12):11520, 2005

7. Sydiskis RJ, Owen DG, Lohr JL, Rosler KH, Blomster RN. The extent of antiviral activity present in a number of plant extracts, Department of Microbiology, University of Maryland, and Baltimore.

8. Agarwal SK, Singh SS, Verma S, Kumar S, The anti fungal activity of anthraquinone derivatives of Rheum emodi, Central Institute of Medicinal and Aromatic Plants, India.2000.

9. Byung Pal Yu, Jeremiah Herlihy and Yuji Ikeno, Effects of long-term aloe ingestion on laboratory rats, Department of Physiology, University of Texas Health Science Center, San Antonio.

10. Visuthikosol V, Chowchuen B, Sukwanarat $Y$, Sriurairatana S, Boonpucknavig V. Thailand Journal Med Assoc Thai. ; 78(8):403-9. 1995

11. Bland, J. Ph.D. Prevention Magazine, Effect of Orally Consumed Aloe Vera Juice in Gastrointestinal Function in Normal Humans; Linus Pauling Institute of Science and Medicine, Palo Alto, C.A. (1985).

12. Fahim MS, Wang M, Contraceptive effect of Aloe barbadensis and zinc acetate, Contraception; 53(4):2316. 1996, USA 